\title{
Circadian Rhythm of Circulating Sclerostin in Healthy Young Men
}

\author{
${ }^{1,2}$ Santosh HS, ${ }^{1}$ Ahluwalia R, ${ }^{1}$ Hamilton A, ${ }^{3}$ Barraclough DL, ${ }^{4}$ Fraser WD, ${ }^{1}$ Vora JP,
}

${ }^{1}$ Department of Diabetes and Endocrinology, Royal Liverpool University Hospital, Prescot Street, Liverpool. L7 8XP. UK, ${ }^{2}$ Department of Endocrinology and Diabetes, Singleton Hospital, Sketty Lane, Sketty, Swansea. SA2 8QA. UK, ${ }^{3}$ Department of Clinical Chemistry, Duncan Building, Royal Liverpool University Hospital, Daulby Street, Liverpool. L69 3GA. UK, ${ }^{4}$ Department of Medicine, Norwich Medical School, University of East Anglia, Norwich. NR4 7TJ. UK

\section{Introduction}

- Sclerostin is a protein predominantly secreted by the osteocytes that has been identified as a physiological inhibitor of bone formation. ${ }^{1}$ It is expressed as a product of the SOST gene translation. By inactivating LRP5, sclerostin inhibits the Wnt signalling pathway and thereby limits bone formation.

-Circadian rhythms have been previously demonstrated for osteotropic hormones like PTH. These rhythms are either truly endogenous or thought to be regulated by calcium and phosphate, but neurological or neurochemical control cannot be dismissed.

-Until recently treatments for osteoporosis have mainly been antiresorptive with intermittent injections of PTH being the only licensed anabolic therapy. Sclerostin inhibition leads to diffuse increase in bone density and may therefore hold promise as another anabolic therapy for osteoporosis. Monoclonal antisclerostin antibodies have been evaluated in trials and show a statistically significant dosedependent increase in bone formation markers ${ }^{2}$.

-The factors controlling the physiology of sclerostin secretion will be of major interest to anyone wishing to establish newer therapeutic options for manipulating bone metabolism to obtain anabolic effects. It is essential to elucidate the physiology of sclerostin secretion both in health and in disease states.

Aim

-A cross-sectional study was undertaken to ascertain whether true endogenous sclerostin circadian rhythmicity exists in healthy individuals

\section{Materials and Methods}

-6 healthy young men were recruited. All patients had bone densitometric evaluation using a Prodigy Oracle Fan-Beam bone densitometer (GE Medical Systems, Giles, Buckinghamshire, UK).

-Blood samples were collected every hour. Samples were centrifuged immediately, and serum/plasma was separated to be frozen at $-70^{\circ} \mathrm{C}$ for later analysis.

-Sclerostin was measured by an enzyme linked immunoassay (Biomedica, Austria). All samples were assayed in duplicates. The intra and inter assay CV\% being $<5 \%$ and $12.3 \%$ respectively.

-Serum calcium, phosphate, creatinine, and albumin were measured by standard procedures on an automated platform (Hitachi 747; Roche Diagnostics, Lewes, UK). Serum Calcium was adjusted for albumin.

-Serum PTH was measured using the Advantage automated assay platform (Nichols Institute, San Juan Capistrano, CA, USA), with a detection limit of $0.5 \mathrm{pmol} / \mathrm{L}$ and intra- and inter assay CVs of $<7 \%$ across the working range.

-1, 25-dihydroxy vitamin $D$ and 25-hydroxy vitamin $D$ were both measured using radioimmunoassay kits (from IDS, Boldon, UK and DiaSorin, Stillwater, MN respectively) after acetonitrile extraction. The intra assay CV was $<9 \%$ and the inter assay CV was $<12 \%$ across the working range for 1, 25-dihydroxy vitamin $\mathrm{D}$, with a detection limit of $15 \mathrm{pmol} / \mathrm{L}$. For the 25-hydroxy vitamin $\mathrm{D}$ assays the intra and inter assay CV's were $<8 \%$ and $<11 \%$ across the working range, with a detection limit of $4 \mathrm{nmol} / \mathrm{L}$.

-Serum concentration of type-I collagen- $\beta$ C-telopeptide (CTX) and procollagen type-I aminoterminal propeptide (PINP) were measured on the Elecsys automated platform, which uses electrochemiluminescence assays (ECLIA; Roche Diagnostics, Lewes, UK). The intra and inter assay CVs for $\beta$ CTX were $<4 \%$ and $<5 \%$, respectively, with a detection limit of $0.01 \mu \mathrm{g} / \mathrm{L}$ and the intra- and inter assay CVs for PINP, were $<2 \%$ and $<2.5 \%$, respectively, across the working range, with a detection limit of $4 \mu \mathrm{g} / \mathrm{L}$.

-IGF-1 was measured with a specific RIA in the presence of a large excess of IGF-2 (Mediagnost, Tübingen, Germany) to block the interference of IGF-binding proteins. Intra- and inter assay CVs were $1.6 \%$ and $6.4 \%$, respectively.

\section{Statistical Analysis}

-Individual and population mean cosinor analysis was performed using CHRONOLAB 3.0 (Universdade de Vigo, Vigo, Spain), a software package for analyzing biological time series by least squares estimation. ${ }^{3}$

•Circadian rhythm parameters evaluated were: 1) midline estimate statistic of rhythm (MESOR), rhythm-adjusted mean; 2) amplitude and 3) acrophase.

-A p value for the rejection of the zero-amplitude (no rhythm) assumption is also determined for each individual series and for the group.

-The onset of sclerostin rise was defined as the time of first occurrence of at least 3 consecutive samples exceeding the mean levels of the sclerostin by more than 1 SD. 5
Results

\begin{tabular}{l|ll}
\hline \multicolumn{3}{|l}{ Table 1: Patient Demographics } \\
\hline 6 Subjects & Mean \pm SD & \multicolumn{1}{c}{ Range } \\
\hline Age (yrs) & $26 \pm 4.1$ & $21-32$ \\
Height (m) & $1.73 \pm 0.07$ & $1.62-1.84$ \\
Weight (kg) & $76.9 \pm 5.7$ & $66.4-83$ \\
T-score Lumbar Spine & $-0.2 \pm 0.9$ & $>-1.0$ \\
T-score Femoral Neck & $-0.5 \pm 0.8$ & $>-1.0$
\end{tabular}

\begin{tabular}{|c|c|c|}
\hline & Mean \pm SD & Range \\
\hline 1-25 OH Vit D (pmol/L) & $86.2 \pm 26.3$ & $43-144$ \\
\hline $25 \mathrm{OH}$ Vit D (nmol/L) & $60.7 \pm 37.5$ & $>50$ \\
\hline IGF 1 (nmol/L) & $148.8 \pm 25.7$ & $34-143$ \\
\hline Creatinine $(\mu \mathrm{mol} / \mathrm{L})$ & $84.8 \pm 16.0$ & $50-130$ \\
\hline Testosterone (nmol/L) & $22.6 \pm 2.9$ & $9-40$ \\
\hline PTH (pmol/L) & $3.7 \pm 0.6$ & $1.1-6.9$ \\
\hline Calcium (mmol/L) & $2.30 \pm 0.11$ & $2.20-2.60$ \\
\hline Phosphate (mmol/L) & $1.13 \pm 0.09$ & $0.70-1.40$ \\
\hline$\beta-C T X(\mu \mathrm{g} / \mathrm{L})$ & $0.29 \pm 0.10$ & $0.10-0.50$ \\
\hline P1NP ( $\mu g / L)$ & $67.7 \pm 35.7$ & $20-76$ \\
\hline
\end{tabular}

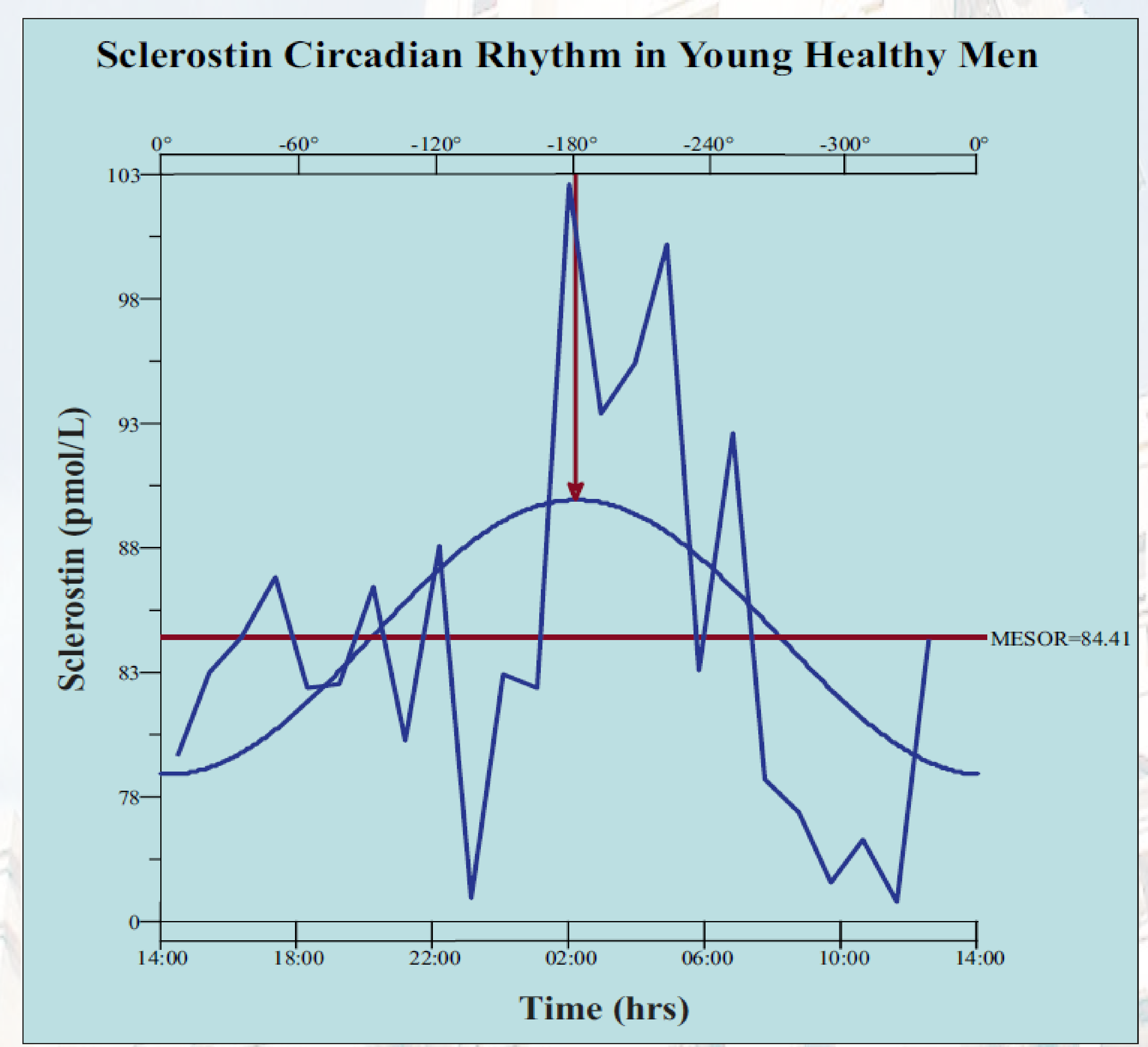

Summary and Conclusions

-Significant circadian rhythm was observed for sclerostin in the study population $(\mathrm{p}=0.028)$.

-The mean sclerostin (MESOR) was $84.4 \pm 1.4 \mathrm{pmol} / \mathrm{L}$.

-Circulating sclerostin demonstrated a nocturnal peak (time of onset 0100h) with concentrations remaining above mean over 4 hours. The sclerostin levels then progressively decline to reach a nadir (at $1000 \mathrm{~h}$ ) the levels remain low throughout the morning till midday (1200h)

- The maximum percentage increase in the sclerostin concentration between $0100 \mathrm{~h}$ and $0700 \mathrm{~h}$ [(value at each time point $-0100 \mathrm{~h}$ value)/ $0100 \mathrm{~h}$ value $\mathrm{x} 100]$ ) was $19.7 \%$

-This is the first demonstration of a definite circadian rhythm in the secretion of sclerostin in young healthy males.

-It may be possible to achieve maximum beneficial effects by altering the prevailing hormone balance by timing the administration of anti-sclerostin therapies prior to the nocturnal rise. 